\title{
The impact of tobacco smoking on treatment choice and efficacy in inflammatory bowel disease
}

\author{
Steven Nicolaides, Abhinav Vasudevan, Tony Long, Daniel van Langenberg \\ Department of Gastroenterology, Eastern Health, Box Hill Hospital, Box Hill, Australia
}

Smoking significantly increases the risk of developing and worsens Crohn's disease (CD), yet protects against the development and reduces the severity of ulcerative colitis. It is less clear whether smoking impacts the efficacy of therapeutics in inflammatory bowel disease (IBD). We review the literature regarding the relationship between smoking and the efficacy of medical and surgical therapy in IBD. Smoking is associated with alterations in thiopurine metabolism and may affect time to disease relapse. The outcomes of anti-tumor necrosis factor therapy in active smokers appear neutral with data lacking for newer biologics. Smoking increases the risk of postoperative recurrence in those requiring resection for CD, likely attributable to perturbations of the gut microbiota although further implications of these for disease onset/progression and treatment efficacy remain unclear. Multiple lifestyle and psychosocial confounders are likely under-recognized cofactors in the association between smoking and IBD. Despite the widely promulgated risks associated with cigarette smoking in CD, more incisive data are required to further elucidate the actual relationship between smoking and disease pathways, while accounting for the several negative cofactors prevalent in smokers which cast uncertainty on the magnitude of the direct effect of smoking on disease pathophysiology and the efficacy of therapy. (Intest Res 2021;19:158-170)

Key Words: Smoking; Tobacco; Inflammatory bowel disease; Crohn disease; Therapeutics

\section{INTRODUCTION}

Smoking is the leading cause for preventable death and accounts for $9 \%$ of the total burden of disease. ${ }^{1}$ With widespread health promotion initiatives there has been a $10 \%$ decrease in smoking rates over the last two decades and up to $75 \%$ of young adults aged between 18 and 24 have never smoked, an increase of $6 \%$ over the last 5 years. ${ }^{2}$

In contrast relatively higher rates of smoking have persisted in patients with inflammatory bowel disease (IBD), in particular Crohn's disease (CD), with prevalence rates of those who have ever smoked ranging between $8.7 \%$ and $64 \%$ in large co-

Received March 6, 2020. Revised May 19, 2020. Accepted May 22, 2020. Correspondence to Steven Nicolaides, Department of Gastroenterology, Eastern Health, Box Hill Hospital, 8 Arnold Street, Box Hill 3128, Australia. Tel: +61-39895-3890, Fax:+61-99822159, E-mail: steven.nicolaides@ easternhealth.org.au horts. ${ }^{3}$ It is well established that smoking significantly increases the risk of developing and worsens the disease course of $\mathrm{CD}$, yet is protective against the development and reduces the severity of ulcerative colitis (UC) ${ }^{4,5}$ However, what is less clear is whether smoking impacts the efficacy of therapeutics in IBD. Given the increased choice and availability of therapies with differing mechanisms of action, it may be surmised that for patients who continue smoking (i.e., failing efforts at cessation as is often encountered) there may be a preferred treatment option given a potential differential impact (positive, negative, or neutral) of smoking on individual treatments. Hence, the aim of this paper was to review the literature regarding the relationship between smoking and the efficacy of medical and surgical therapies in IBD, with particular reference to $\mathrm{CD}$. A proposed algorithm for the approach to the management of IBD in smokers is proposed based on this review (Fig. 1). 


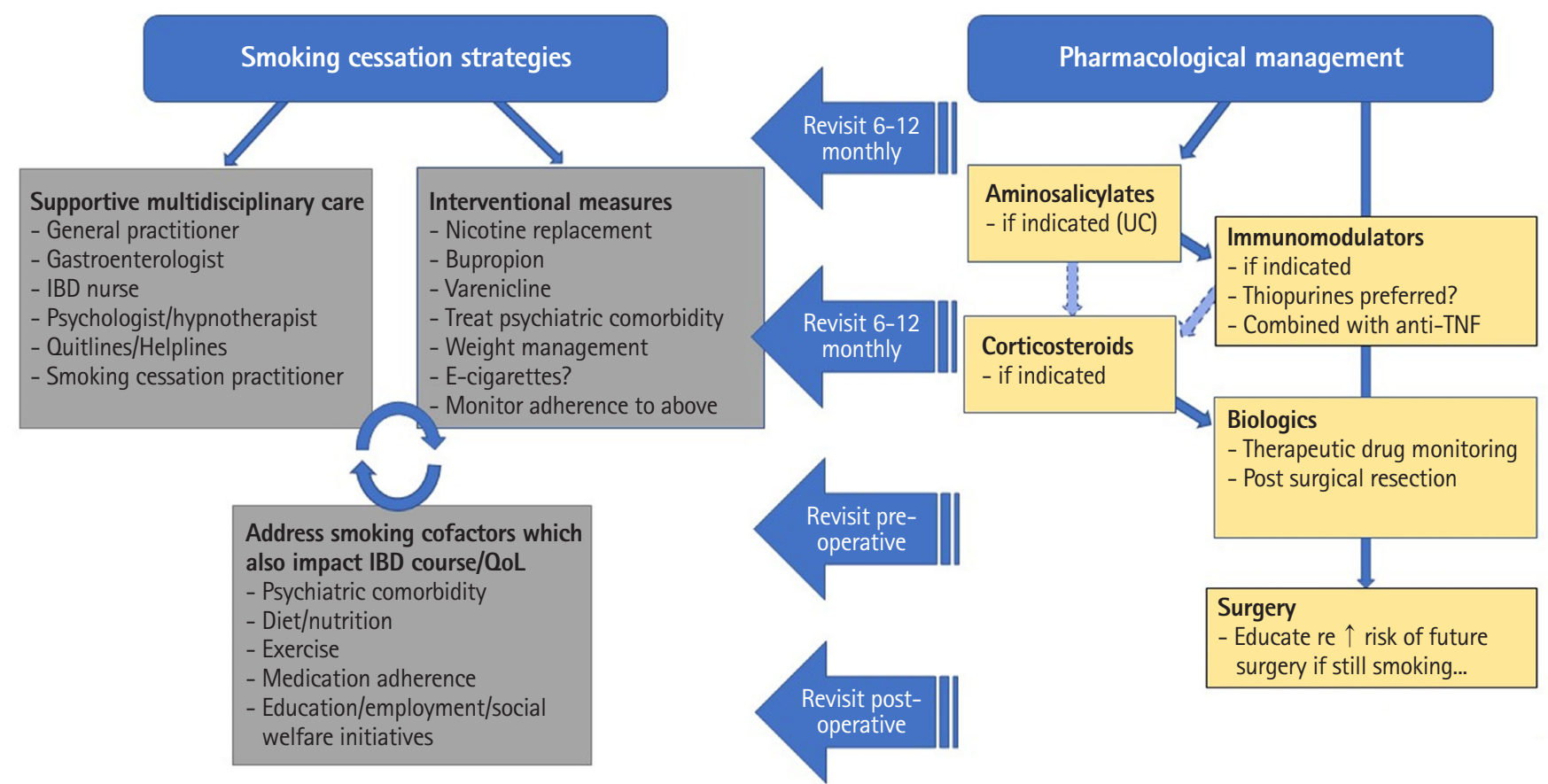

Fig. 1. Algorithm for approach to management of inflammatory bowel disease (IBD) in smokers. QoL, quality of life; UC, ulcerative colitis; TNF, tumor necrosis factor.

\section{METHODS}

A comprehensive review of the English literature for relevant articles from 1946 until April 2019 was performed using Medline (Ovid host), Embase, and the Cochrane central library. The search strategy was designed and conducted by experienced medical library staff following discussion with the study investigators. The following key words and Medical Subject Headings (MeSH) were used either alone or in combination "inflammatory bowel, "disease," "crohn*” "ulcerative," "nicotine" "tobacco," "smoking," "smoking cessation”, "smoking reduction", "tobacco use cessation", "electronic nicotine delivery systems", "tobacco products", "smoker*”, “vaping", "cigarette*”, "steroid*”, “azathioprine,", "biologic*”, “infliximab”, "thiopurine", "dilatation”, "tumor," “tumour", "necrosis factor”, "mesalamine”, “5 ASA”, “5 aminosal", "relapse*”, "recurr*”, "outcome*”, "remission", "relapse", "recurr*", "outcome*" and "remission." The title and abstract for identified studies were evaluated and studies that did not relate to the research questions of interest were excluded. Additional studies were identified by a separate search of the reference list of identified relevant studies and review articles. A total of 354 articles were identified from the initial search.

\section{THE RELATIONSHIP BETWEEN CONCURRENT SMOKING AND OUTCOMES OF SPECIFIC THER- APEUTICS IN IBD}

\section{Aminosalicylates and Immunomodulators}

No studies were identified that explored a link between efficacy of aminosalicylates and smoking status. The immunomodulators azathioprine and 6-mercaptopurine are purine analogues metabolized via a well described enzymatic pathway, with the 6-thioguanine nucleotides (TGN) determined to be the active metabolite. Multiple studies have shown an association between smoking and higher 6-TGN levels. ${ }^{6,7}$ In a retrospective study of 132 patients, Poon et al. ${ }^{7}$ examined the influence of multiple clinical variables on 6-TGN levels and found smokers to have higher levels in comparison with non-/exsmokers (odds ratio $[\mathrm{OR}], 1.43 ; 95 \%$ confidence interval $[\mathrm{CI}]$, $1.02-2.02 ; P=0.041)$. They did not report on any clinical markers of disease response. The mechanism for the increase in 6-TGN levels is unclear particularly given that previous studies have been demonstrated that smoking may upregulate xanthine oxidase transcription and activity. ${ }^{8}$

Moreover, a few studies have reported on the potential impact of smoking on the efficacy of thiopurines. In a retrospective study of 163 patients with steroid-dependent IBD it was 
found that active smoking did not adversely impact rates of steroid-free remission achieved with thiopurine therapy. ${ }^{9}$ This effect persisted across both the CD and UC cohorts, though among the CD subgroup active smoking resulted in a higher probability of requiring new steroid course during follow-up ( $53 \%$ vs. $23 \%, P=0.028$ ). In addition, active smoking was the only independent factor associated with a higher risk of discontinuation of thiopurine therapy because of adverse effects. ${ }^{9}$

Thiopurines also appear effective in preventing postoperative recurrence in CD. The TOPPIC study, a randomized control trial (RCT) comparing mercaptopurine and placebo in preventing postoperative recurrence after surgical resection, found that smokers treated with mercaptopurine were less likely to have disease recurrence at 3 years compared to smokers treated with placebo (hazard ratio [HR], 0.13; 95\% CI, 0.040.46). ${ }^{10}$ Conversely there was no difference in recurrence rates between the mercaptopurine and placebo groups in nonsmokers. Moreover, there was no disparity in reoperation rates as per a retrospective analysis of smoking and azathioprine between smokers and non-smokers. ${ }^{11}$ Hence, these findings potentially suggest that despite concurrent smoking, commencing thiopurines early after surgical resection in smokers remains an effective strategy. However, less favorable results have been found in studies which assessed short term response. The POCER (Post-operative Crohn's Endoscopic Recurrence) study, a prospective study assessing postoperative recurrence in $\mathrm{CD}$, found that regardless of therapy with either metronidazole, azathioprine or adalimumab, smoking significantly increased the risk of endoscopic disease recurrence at 18 months. $^{12}$

It has also been suggested that smoking prolongs the time to recurrence following thiopurine cessation. In a retrospective analysis of $141 \mathrm{CD}$ patients in remission treated with azathioprine for a period of $98.4 \pm 5.7$ months, non-smoking was in fact associated with earlier relapse $(\mathrm{OR}, 2.8 ; P=0.006){ }^{13}$ These findings were also demonstrated by a GETAID study which found that smoking was actually protective of relapse in those treated with azathioprine for extended durations ( $>36$ months).$^{14}$ The underlying mechanistic explanation for this is unclear, but might possibly relate to a prolongation of effect of smoking on azathioprine metabolism, leading to persistent activity well beyond cessation of therapy.

Methotrexate, an antimetabolite that influences multiple enzymes utilizing folate for the synthesis of purines and pyrimidines, has been increasingly used in the management of $\mathrm{CD}$ in recent decades. One retrospective study assessed the efficacy of antimetabolites including methotrexate in maintaining remission of $\mathrm{CD}$ incorporated smoking status in the analysis. Within the cohort of 92 patients where anti-tumor necrosis factor (TNF) therapy was ceased following remission and maintenance antimetabolite therapy continued, active smoking was identified as a risk factor for relapse (HR, 1.91; 95\% CI, $1.11-3.27 ; P=0.02$ ) as well as perianal disease. ${ }^{15}$ The potential interaction between methotrexate efficacy and smoking status has been further delineated in rheumatological studies. For instance, in a review of the Swedish Rheumatology Register of 1,430 patients treated with methotrexate, current smokers were less likely to achieve a "good response" at 3 months and at later review date post commencing methotrexate ${ }^{16}$ In a further study assessing the factors associated with inadequate response to methotrexate in 108 patients with rheumatoid arthritis previously naïve to disease modifying antirheumatic drugs, showed that current smoking and alcohol consumption at baseline were predictive of inadequate response to therapy. ${ }^{17}$

\section{Anti-TNF Agents}

Traditional dogma, anecdotal experience and some studies have linked active smoking with an attenuated clinical response to anti-TNF therapy in IBD, particularly CD, compared with non-smokers. ${ }^{18,19}$ There are multiple potential mechanisms at play. Smokers administered infliximab for CD exhibit lower median trough levels than non-smokers $(3.4 \mathrm{mg} / \mathrm{L}$ vs. $13.3 \mathrm{mg} / \mathrm{L}){ }^{20}{ }^{20}$ In addition, anti-infliximab antibodies were found to be significantly higher in smokers than in non-smokers, implying a diminished response to infliximab in CD based on pharmacokinetic effects. ${ }^{20}$

From a pharmacodynamic perspective, smokers with rheumatoid arthritis have been found to exhibit increased TNF- $\alpha$ release from stimulated Tymphocytes isolated from peripheral blood samples. $^{21}$

However, despite these findings, the overall impact of concurrent smoking on the efficacy of anti-TNF agents remains somewhat unclear. A systematic review by Narula and Fedor$\mathrm{ak}^{22}$ specifically analyzed smoking and its impact on the efficacy of infliximab across 10 studies comprising a total of 1,779 patients. Eight of these showed no difference in response and relapse rates for both luminal and fistulizing CD although the remaining 2 studies demonstrated that smokers had reduced early response rates and shorter time to relapse.

Also, a more recent meta-analysis examining the impact of smoking on early response to infliximab by Inamdar et al. $^{23}$ 
analyzed 8 studies, comprising 1,658 patients. Most were prospective studies and assessed both luminal and fistulizing CD. Again, no clear association was found between smoking and response to infliximab, with a relative risk (RR) of 0.99 (95\% CI, 0.88-1.11; $P=0.0143$ ) for infliximab response among smokers. The limitations of this meta-analysis were similar to those encountered by Narula et al. (indeed, both included similar studies). These methodological limitations included the lack of consistency and variation in the definition of smoking, both in terms of duration and quantity of smoking. There were also varied methods in data collection both prospective and retrospectively acquired and application of different symptom-based disease indices (e.g., Harvey Bradshaw Index vs. Crohn's Disease Activity Index).

\section{Non-Anti-TNF Biologics and Small Molecules}

In one of the first real world efficacy studies of vedolizumab in $\mathrm{CD}$, a reduced likelihood of achieving clinical remission after 12 months of vedolizumab was demonstrated in current or former smokers (HR, 0.47; 95\% CI, 0.25-0.89) ${ }^{24}$ Furthermore, with reference to its fellow anti- $\alpha 4$ integrin molecule natalizumab, smoking was associated with a higher likelihood of anti-natalizumab antibody development in multiple sclerosis patients and smoking was associated with higher risk of relapse in multiple sclerosis patients on natalizumab. ${ }^{25,26}$

We were unable to identify any studies that have examined the relationship between response to ustekinumab in IBD while accounting for smoking status. One study in psoriasis showed no difference in therapeutic response between smokers and non-smokers when assessed at 3-, 6-, and 12-month timepoints of ustekinumab treatment. ${ }^{27}$ However another study found that those deemed to be "insufficient" responders were more likely to have a smoking habit of $>20$ cigarettes per day. ${ }^{28}$ Interestingly, ustekinumab appears to be the most effective option for treatment of anti-TNF induced psoriasiform skin lesions in IBD, which have been characterized by the presence Th17 and Thl cell infiltrates and higher concentrations of interleukin (IL)-17A-expressing T cells. Smoking (OR, 4.24; 95\% CI, 1.6-13.6) was found to be the strongest predictor of these skin lesions, hence one might extrapolate that ustekinumab could be a preferred biologic option in current smokers as at least in a subset of patients, smoking may promote inflammatory pathways amenable to ustekinumab's anti-IL-12/IL-23 activity, though this obviously requires further confirmation both from a mechanistic and clinical perspective. ${ }^{29}$

Tofacitinib, an inhibitor of Janus kinase 1 and 3, has recently been shown to be effective in the management of patients with UC. ${ }^{30}$ To date there are no studies which have examined the impact smoking status has on the efficacy of tofacitinib in the treatment of UC. In the rheumatological cohort where tofacitinib is used mainly in the treatment of rheumatoid arthritis there is poor quality evidence mainly in abstract form that suggests smoking has no deleterious effect on response to tofacitinib. $^{31}$

Recently there has been safety concerns over the use of tofacitinib at the higher dose of $10 \mathrm{mg}$ twice daily identified in a post-marketing safety trial. ${ }^{32}$ However, a recent post-hoc analysis of trials of tofacitinib in patients with UC found there was no significant difference in the rates of deep vein thrombosis or pulmonary embolism in patients treated with tofacitinib compared to those on no treatment. ${ }^{33}$ Nevertheless, given the potentially increased risk of thrombosis and venous thromboembolism in smokers, caution is advisable prior to commencing tofacitinib in smokers with UC. Finally, the risk of herpes zoster infection with tofacitinib appears augmented by current smoking status. ${ }^{34}$

\section{Fecal Microbiota Transplant}

The intestinal microbiota is believed to play an integral role in the pathogenesis of IBD. ${ }^{35}$ This has naturally piqued interest in the role of fecal microbiota transfer (FMT) in the management of IBD. A recent systematic review and meta-analysis concluded that FMT was effective in achieving induction of remission in patients with UC. ${ }^{36}$ In addition, a recent pilot study highlighted that once remission of UC was achieved with FMT, 8 weekly FMT was effective maintenance treatment out to 48 weeks. ${ }^{37}$ Furthermore, the utility of FMT in CD holds early promise, with remission rates of over $50 \%$ reported in 4 case reports and 7 prospective uncontrolled studies. ${ }^{36}$

However as yet, no studies to date have attempted to assess whether smoking is an influential cofactor in FMT treatment outcomes. Nevertheless, smoking has been shown to potentially affect the gut microbiota by altering immune-microbial interactions within the gut as well as mucus production. ${ }^{38}$ For instance, gut microbial diversity has been shown to be altered by smoking status, with higher levels of Bacteroides spp one of the most striking findings, which then appears to decrease following smoking cessation. ${ }^{39}$

\section{The Impact of Smoking Status on Surgical/ Endoscopic Intervention(s)}

While there has been significant advancement in the medical 
therapy of IBD a significant proportion of patients fail to achieve durable remission and require surgical resection. ${ }^{40}$ Particularly in CD, a large number of patients will experience disease recurrence proximal to the site of surgical anastomosis, of which up to a third will be symptomatic and a small but significant proportion will require a subsequent resection. ${ }^{41} \mathrm{In}$ the POCER study, endoscopic recurrence was prospectively assessed following surgical resection. Smoking was found to be a strong risk factor for postoperative recurrence with recurrence rates of $67 \%$ versus $49 \%$ (OR of 2.4 ), despite optimization of medical therapy. ${ }^{12}$ A risk of similar magnitude was also demonstrated in a meta-analysis of observational studies which demonstrated a 2-fold risk of clinical recurrence and reoperation rates with smoking. ${ }^{42}$

Another subsequent meta-analysis assessed the impact of smoking on the need for a subsequent first surgical resection in both CD and UC. Again, it was found that smoking increased the risk of surgical intervention in $\mathrm{CD}$, though the risk was only increased in current and not former smokers. Conversely, smoking was not associated with increased rates of colectomy in patients with UC. ${ }^{43}$ Importantly, this meta-analysis included historical data where biologics were either not yet established or emerging, so it is impossible to deduce the impact of contemporary medical therapies on the risk of surgical resection in this context.

Endoscopic balloon dilatation of stricturing CD is an effective, alternative to surgery in selected patients and has been shown effective for alleviating obstructive symptoms and avoiding surgery, with rates of surgical resection post dilatation of $30 \%$ after 5 years. ${ }^{44}$ While a short stricture length and absence of ulceration have been characterized as favorable prognostic factors, conversely smoking appears to double the risk of future stricture recurrence and the need for further intervention. ${ }^{44}$

\section{IS SMOKING PURELY TO BLAME?}

\section{Direct Toxic Effects of Cigarette Smoke on Intestinal Milieu/Autoimmunity}

While there is little doubt, as this review has already demonstrated, that smoking is detrimental to treatment outcomes in IBD based on a multitude of observational data, there are remarkably few studies that have explored the underlying mechanisms of which and how particular constituents of tobacco smoking affect the intestinal milieu and/or relevant immune-inflammatory pathways.
The pathogenesis and mechanisms of how smoking leads to many chronic diseases such as chronic obstructive airway disease and cardiovascular disease has been well established. ${ }^{45-47}$ In IBD, smoking has been shown to exert opposite effects on UC and CD. Yet the actual components of cigarette smoke that interact with the intestinal mucosa, immune cells and microbiota leading to and/or influencing this disease state remain elusive. This is largely due to the large number of toxic substances that are generated through combustion releasing $>5,000$ compounds, a significant proportion of which are ingested via the gastrointestinal (GI) tract. ${ }^{48}$ Also, intestinal function is impaired by the particulate matter consumed in cigarette smoke with alterations in intestinal mucus secretion, mucosal microcirculation and mucosal repair processes. ${ }^{49}$

Moreover, at a cellular level cigarette smoke has been shown to affect the dendritic cell phenotypes by way of alteration in molecular and receptor function, with differential impacts in UC compared with CD samples when exposed to cigarette smoke. ${ }^{50}$ Alternate effects on dendritic cells have been demonstrated through exposure to carbon monoxide (CO) in cigarette smoke. $\mathrm{CO}$ prevents the maturation of dendritic cells and lowers the production of pro-inflammatory cytokines and the proliferation of effector T cells, whereas it stimulates the secretion of the anti-inflammatory cytokine IL-10 which may explain the beneficial effects in UC. ${ }^{51}$ Reasons as to why this anti-inflammatory effect predominates in UC as opposed to $\mathrm{CD}$ remain uncertain, yet further differences in cytokine levels have been elucidated, with reduced IL-1 $\beta$ and IL-8 levels in smokers with UC and reduced IL-8 for smokers with CD. ${ }^{52}$

Further to the effects on immune cellular function, one animal study demonstrated that cigarette smoking triggers colitis in mice mediated by CD4+ interferon- $\gamma+$ T cells. ${ }^{53}$ Studies of peripheral blood lymphocytes have also shown modified telomerase expression in smokers with $\mathrm{CD}^{54}$

Another potential explanation for cigarette smoking's role in the risk of development of IBD may be due to influences on gene expression. Studies of sibling pairs with IBD have shown that smokers tend to develop CD and non-smokers are more likely to develop UC. ${ }^{55}$ Genetic studies have identified that the genes RNF138, MT2A, and STEAP3 are up-regulated in smokers with CD, highlighting a potential link between smoking, gene expression and disease development. ${ }^{56}$

As described previously the composition of gut microbiota has been implicated in the development and course of IBD. A few studies have evaluated whether the gut microbiota might be implicated in the differing influence of cigarette smoke on 
UC and CD. Opstelten et al. ${ }^{57}$ examined gut microbial diversity in fecal samples from patients with CD and found that species diversity was significantly reduced in smokers, with reductions in the species Collinsella, Enterorhabdus, and Gordonibacter in particular. Others have identified significantly higher amounts of Bacteroides and Prevotella in smokers with CD compared to non-smokers. ${ }^{58}$

\section{The Interrelationship of Smoking and Other Lifestyle Factors Which May Also Negatively Impact Treatment Outcomes in IBD}

Contemporary data have demonstrated shifting trends of smoking within the IBD cohort that is dependent on geographical and socioeconomic factors. While the rates of CD have remained stable in developed nations across Europe in the 21st century, the proportion of non-smokers has increased. The opposite is true within developing nations across Asia for instance, where rates of CD are increasing and non-smoking rates have decreased. ${ }^{3}$

These geographic factors illustrate the complexities in a chronic disease entity like IBD, with a multifactorial etiopathogenesis and multiple, relatively weak predictive associations of disease prognosis, in assessing whether a variable such as smoking status truly has an independent, significant impact on disease course and/or treatment outcomes. Furthermore, most of the data assessing cigarette smoking's effect on IBD and treatments are primarily from uncontrolled observational studies where it is difficult to account for, much less eliminate, the effects of residual confounders. With regards to smoking status, the list of potential confounders which may impact any association between smoking and adverse outcomes in IBD are legion as depicted in Fig. 2.

For instance, at least among the general population, those who smoke are also less likely to engage in other health-seeking behaviors such as consuming a healthier diet and engaging in regular physical activity. ${ }^{59}$ Yet exercise may have a protective role in the development and course of IBD. The recent meta-analysis by Engels et al. ${ }^{60}$ examined 10 studies from 1990 to 2015 and found that high levels of exercise correlated with a reduced risk (RR, 0.63; 95\% CI, 0.50-0.79) of CD in comparison with those with low exercise levels. Moreover, in a large prospective study of IBD patients in remission (1,308 CD and $549 \mathrm{UC} /$ indeterminate colitis), higher levels of physical activity were associated with lower rates of active disease of CD (RR, 0.72; 95\% CI, 0.55-0.94). ${ }^{61}$ A similar association was found in UC/indeterminate colitis however the results were not statistically significant (RR, 0.78 ; 95\% CI, 0.54-1.13). Multiple studies have also shown that exercise improves disease related quality of life in IBD patients. ${ }^{62,63}$

Furthermore, active smoking status has been linked with consumption of high energy (lower quality) diets, exemplifying the so-called "Western diet" which in turn has been implicated in the development of IBD. ${ }^{64-66}$ Moreover, observational studies have shown that the risk of developing CD was reduced by a diet high in fruit fiber and to a lesser degree vegetable fiber. ${ }^{67,68}$ Indeed, recent interventional dietary studies have shown promise in improved disease control in CD, such as the CD exclusion diet ${ }^{69}$ Yet conversely, current smoking status has been associated with lower fruit and vegetable intake, even when controlling for gender and socioeconomic group. ${ }^{70}$

Moreover, there are considerable data supporting the notion that smokers typically exhibit lower rates of treatment ad-

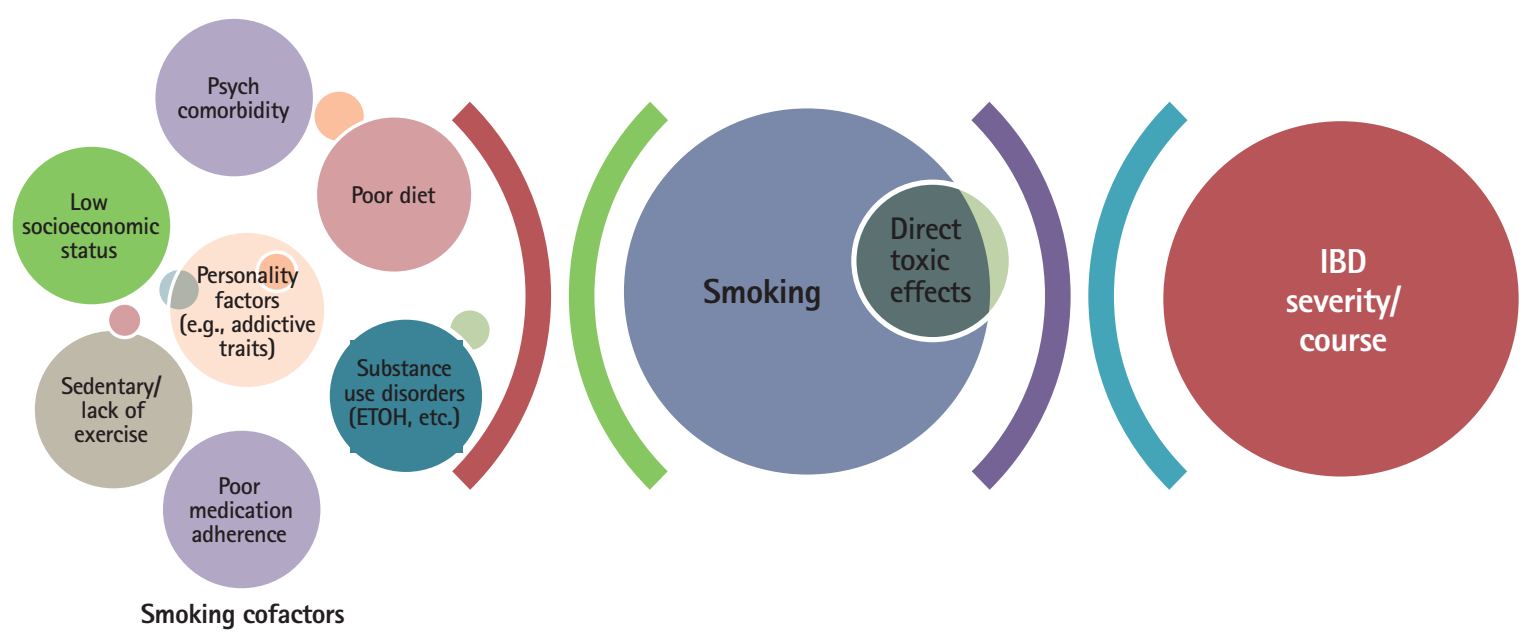

Fig. 2. Smoking cofactors. ETOH, ethanol; IBD, inflammatory bowel disease. 
herence than non-smokers. For instance in a large employer claims database study, current smokers were found to have significantly lower rates of compliance with cancer screening programs as well as lower medication adherence for multiple chronic conditions, findings which persisted even with adjustment for age, sex, ethnicity, income level, and educational achievement. ${ }^{71}$ Indeed also in IBD, current smoking was associated with poor adherence to oral aminosalicylate therapy in a Korean study (OR of 4.1), nonadherence to anti-TNF therapies (both infliximab and adalimumab) in another study, and higher rates of nonadherence to (any) medications in women with IBD during pregnancy was demonstrated in smokers in the study by Nielsen et al. ${ }^{72-74}$

The link between smoking status and psychiatric disorders is also highly pertinent to IBD where, as in other chronic disease states, rates of comorbid anxiety and depression for instance are far higher than the general population. ${ }^{75}$ In turn, psychiatric comorbidity in IBD has been independently associated with increased disease activity, poorer disease outcomes (e.g., increased healthcare utilization) thus poorer quality of life. ${ }^{76-78}$ In addition, smoking has been associated with higher rates of opioid dependence, alcohol dependence and other substance use disorders. These cofactors again may adversely impact on disease course and treatment adherence in IBD. ${ }^{79-81}$

Hence, one might postulate that given the potentially large effect size of the aforementioned factors associated with both smoking status and IBD, that the magnitude of any direct mechanistic or toxic effect of smoking constituents on the intestinal milieu or treatment(s) might in fact be dwarfed by these cofactors, as represented in Fig. 2.

\section{The Role of Unconscious and Other Biases Which May Additionally Impact Treatment Outcomes in Smokers}

In addition to the multiple confounding factors above inexorably linked with smoking status and the overall impact on IBD and other chronic diseases, clinicians need to be cogniscent of unconscious (or overt) bias in relation to smokers, who have increasingly become somewhat marginalized in the 21st century. This bias has the potential to then only further compromise treatment outcomes in smokers. A clinician or health payer can easily depict smoking-related disease as self-inflicted, similar to alcohol and liver disease or poor diet and obesity and thus punitively restrict access to certain treatment options for these patients accordingly. This has the potential to result in a reservation to prescribing actively smoking patients dose intensification of biologics for secondary loss of response, given the expense and resource implications, especially if the individual is unable or unwilling to quit smoking. Yet this raises ethical concerns and ultimately in a universal or user-pays healthcare setting, this is perhaps inappropriate. For example, one would not refuse emergency surgery for a motor vehicle accident victim on the basis that they were not wearing a seat belt. Unless health payers adopt a more utilitarian approach to smoking and legislate accordingly, it remains incumbent on clinicians to provide high quality, consistent medical care to all individuals and smoking is just one of many unhealthy behaviors that humans engage in, often despite the risks. ${ }^{82}$

\section{Challenges and Failures of Smoking Cessation Efforts: Should E-Cigarettes Be Promoted as a Pragmatic Alternative?}

In addition to the potential biases as described above, there is the potential for non-smokers and/or clinicians to underestimate the difficulties of achieving durable smoking cessation.

Despite the wide range of therapies available and evaluated for smoking cessation, including non-pharmacological (e.g., counselling, cognitive-behavioral therapy, hypnotherapy) and pharmacological therapies (including nicotine replacement therapy [NRT], varenicline and bupropion) ${ }_{,}^{83}$ the reality is that durable cessation rates remain low, including in patients with smoking-affected chronic diseases. For instance, amongst all adult smokers in the United States in 2015, only $7.4 \%$ were successful in smoking cessation. ${ }^{84}$ Success rates in the IBD population are particularly hampered by lack of access to support services with over $90 \%$ of smokers with IBD having never received any support to cease smoking and of those who received support only $4 \%$ were counselled by their primary physician. ${ }^{85}$

One controversial yet pragmatic option in the setting of unsuccessful cessation is e-cigarettes. Compared to conventional combustible cigarettes, e-cigarettes appear to in some respects pose less health risks. The lifetime risk of cancer from using ecigarettes is $0.4 \%$ of that of cigarettes. ${ }^{86}$ Further to this a Cochrane review in 2016 found that no serious adverse effects have been detected in e-cigarette use. ${ }^{87}$ The most common side effects of e-cigarettes are mouth and throat irritations which resolve over time. Public Health England has promoted the use of e-cigarettes as a tool for smoking cessation and maintains that e-cigarettes are $95 \%$ less harmful than cigarettes. ${ }^{88}$

Hajek et al. ${ }^{89}$ demonstrated in a 2019 RCT of 886 partici- 
pants that e-cigarettes are more effective for smoking cessation than NRT, when both were combined with behavioral therapy. The 1-year abstinence rate in the e-cigarette group was $18.0 \%$ compared with $9.9 \%$ in the NRT group (RR, 1.83; 95\% CI, $1.30-2.58 ; P<0.001)$. Prior to this study, numerous smaller RCTs found modest associations between e-cigarette use and smoking cessation and other prospective observational studies found mixed results. ${ }^{90-93}$

An issue that has plagued the use of e-cigarettes are the lack of regulations to control the quality and composition of ingredients used in e-cigarettes. Furthermore, the aerosols generated by these products typically contain a variety of substances including but not limited to nicotine, flavorings, additives and tetrahydrocannabinol (THC).$^{94}$ It is no surprise that recent studies have raised concerns about other potential serious side effects of the use of e-cigarettes. These include case reports of traumatic injury as a result of e-cigarette explosion as well as the emerging syndrome of e-cigarette, or vaping, product use-associated lung injury (EVALI). ${ }^{94-96}$ The emerging clinical syndrome of EVALI is of particular concern with the Center for Disease Control and Prevention reporting 60 cases that have ultimately resulted in the death of those affected..$^{94} \mathrm{It}$ is important that clinicians are aware of these significant risks when counselling patients who are either currently using ecigarettes or considering its use a substitute for smoking cigarettes.

The effects of e-cigarettes on the disease course and response to therapy in IBD remains unclear. In a 2018 pilot study, Stewart et al..$^{97}$ found as expected, that compared to controls, tobacco smokers had significantly altered intestinal bacterial profiles, yet e-cigarette users had similar oral and intestinal microbiota as controls. Further research is needed to examine the impact of e-cigarettes on the GI system and its diseases.

\section{DISCUSSION}

In real world practice, smoking cessation, similar to endoscopic or histologic healing may be seen as an aspirational goal which, despite clinicians and patients' considerable efforts, unfortunately may be durably achieved in only a minority of cases. Hence, as demonstrated in this review, management for patients with IBD should engender a holistic approach which addresses not only smoking cessation but also multiple other lifestyle cofactors such as diet, exercise and mental health plus optimization of medical therapeutics.
This review has reiterated that cigarette smoking is one of the strongest predictors of poorer prognosis and to a somewhat lesser extent, response to treatment in CD. There is evidence to suggest that smoking may promote antibody development to multiple biologics, thus confer risk of loss of response accordingly and increase the risk of postoperative recurrence and subsequent surgeries in CD. However further data supporting a detrimental impact on treatment outcomes per se are surprisingly sparse. For instance, 2 large meta-analyses were unable to depict an effect of smoking status on response to infliximab, ${ }^{22,23}$ and smoking, if anything, may have a positive impact on thiopurine metabolism and related treatment response.

Therefore, if a patient is unable to achieve smoking cessation with appropriate support, the available evidence implies that current smokers with CD should in fact be treated more aggressively with biologic and/or thiopurine therapy in an attempt to mitigate future disease risk, especially future surgeries. This is in contrast to the alternative, somewhat biased approach of withholding treatment escalation in smokers until they quit, in the hope that this will enhance disease outcomes thereafter. But this process may take years and in reality, is statistically unlikely to happen, leading to problematic treatment delays and potentially further IBD complications. Instead, there is a clear need to aggressively optimize medical therapy which represents one potentially modifiable variable to offset the multiplicity of negative cofactors associated with active smoking status that likely have a detrimental role in the course and severity of IBD, yet are not so easily modifiable, including socioeconomic and educational status, dietary behaviors and medication (non)adherence.

Future directions of research into smoking and IBD should also explore the potential for e-cigarettes to aid smoking cessation and in the interim, minimize tobacco consumption in IBD patients. There is evidence for growing popularity of e-cigarette use in the general and in the IBD population, the latter with rates of approximately $4 \% .^{98}$ Although there are current concerns around safety and lack of industry regulation, if these can be addressed, this may hold significant promise for refractory smokers in particular.

\section{CONCLUSIONS}

Despite the well-known risks associated with cigarette smoking in CD, we require more sophisticated "big data" type studies to further elucidate the true relationship between smoking 
and disease pathways, while accounting for the several negative cofactors found in active smokers which cast significant uncertainty on the magnitude of the direct effect of smoking on disease pathophysiology. There are also remarkably little data on smoking cessation strategies and outcomes specifically in IBD populations.

Finally, smokers with CD represent a challenge to clinicians and can be a source of frustration given their adverse disease prognosis and perceived poor health behavior. Nevertheless, there is a shared responsibility to achieve health by both patients and clinicians. Smoking cessation strategies should therefore be repeatedly offered and promoted by clinicians with success more likely to be achieved in a well-resourced, multidisciplinary, holistically-minded healthcare setting, along with considerable patience.

\section{ADDITIONAL INFORMATION}

\section{Funding Source}

The authors received no financial support for the research, authorship, and/or publication of this article.

\section{Conflict of Interest}

No potential conflict of interest relevant to this article was reported.

\section{Author Contribution}

Conceptualization: Nicolaides S, van Langenberg D. Methodology: Nicolaides S, Vasudevan A, van Langenberg D. Visualization: Nicolaides S, van Langenberg D. Writing - original draft: Nicolaides S, Long T. Writing - review and editing: Nicolaides S, Vasudevan A, van Langenberg D, Long T. Approval of final manuscript: all authors.

\section{ORCID}

Nicolaides S

Vasudevan A

Long $\mathrm{T}$

van Langenberg D

https://orcid.org/0000-0003-1726-497X https://orcid.org/0000-0001-5026-9014 https://orcid.org/0000-0003-0091-1739 https://orcid.org/0000-0003-3662-6307

\section{REFERENCES}

1. National Center for Chronic Disease Prevention and Health Promotion (US) Office on Smoking and Health. The health consequences of smoking-50 years of progress: a report of the surgeon general. Atlanta: Centers for Disease Control and
Prevention (US), 2014.

2. Australian Institute of Health and Welfare (AIHW). Australia's health 2018. Australia's health series no. 16. AUS 221. Canberra: AIHW, 2018.

3. Thomas T, Chandan JS, Li VSW, et al. Global smoking trends in inflammatory bowel disease: a systematic review of inception cohorts. PLoS One 2019;14:e221961.

4. Mahid SS, Minor KS, Soto RE, Hornung CA, Galandiuk S. Smoking and inflammatory bowel disease: a meta-analysis. Mayo Clin Proc 2006;81:1462-1471.

5. Boyko EJ, Perera DR, Koepsell TD, Keane EM, Inui TS. Effects of cigarette smoking on the clinical course of ulcerative colitis. Scand J Gastroenterol 1988;23:1147-1152.

6. Meijer B, Seinen ML, Hosman T, et al. High inter-individual variability of serum xanthine oxidoreductase activity in IBD patients. Nucleosides Nucleotides Nucleic Acids 2018;37:317323.

7. Poon SS, Asher R, Jackson R, et al. Body mass index and smoking affect thioguanine nucleotide levels in inflammatory bowel disease. J Crohns Colitis 2015;9:640-646.

8. Kayyali US, Budhiraja R, Pennella CM, et al. Upregulation of xanthine oxidase by tobacco smoke condensate in pulmonary endothelial cells. Toxicol Appl Pharmacol 2003;188:5968.

9. Domènech E, Carrión S, Garcia-Planella E, et al. Smoking status and response to thiopurines in steroid-dependent inflammatory bowel disease. Inflamm Bowel Dis 2011;17:971-975.

10. Mowat C, Arnott I, Cahill A, et al. Mercaptopurine versus placebo to prevent recurrence of Crohn's disease after surgical resection (TOPPIC): a multicentre, double-blind, randomised controlled trial. Lancet Gastroenterol Hepatol 2016;1:273-282.

11. Lakatos PL, Szamosi T, Czegledi Z, et al. S1143 Azathioprine/ biological therapy does prevent surgery but not reoperation in smokers with Crohn's disease. Gastroenterol 2009;136:A199.

12. De Cruz P, Kamm MA, Hamilton AL, et al. Crohn's disease management after intestinal resection: a randomised trial. Lancet 2015;385:1406-1417.

13. Sokol H, Seksik P, Nion-Larmurier I, Vienne A, Beaugerie L, Cosnes J. Current smoking, not duration of remission, delays Crohn's disease relapse following azathioprine withdrawal. Inflamm Bowel Dis 2010;16:362-363.

14. Treton X, Bouhnik Y, Mary JY, et al. Azathioprine withdrawal in patients with Crohn's disease maintained on prolonged remission: a high risk of relapse. Clin Gastroenterol Hepatol 2009; 7:80-85. 
15. Chauvin A, Le Thuaut A, Belhassan M, et al. Infliximab as a bridge to remission maintained by antimetabolite therapy in Crohn's disease: a retrospective study. Dig Liver Dis 2014;46: 695-700.

16. Saevarsdottir S, Wedrén S, Seddighzadeh M, et al. Patients with early rheumatoid arthritis who smoke are less likely to respond to treatment with methotrexate and tumor necrosis factor inhibitors: observations from the Epidemiological Investigation of Rheumatoid Arthritis and the Swedish Rheumatology Register cohorts. Arthritis Rheum 2011;63:26-36.

17. Teitsma XM, Jacobs JWG, Welsing PMJ, et al. Inadequate response to treat-to-target methotrexate therapy in patients with new-onset rheumatoid arthritis: development and validation of clinical predictors. Ann Rheum Dis 2018;77:12611267.

18. Parsi MA, Achkar JP, Richardson S, et al. Predictors of response to infliximab in patients with Crohn's disease. Gastroenterology 2002;123:707-713.

19. Arnott ID, McNeill G, Satsangi J. An analysis of factors influencing short-term and sustained response to infliximab treatment for Crohn's disease. Aliment Pharmacol Ther 2003;17: 1451-1457.

20. Kong JY, Bundell CS, Pawlik J, Hollingsworth PN, Forbes GM. Smoking is associated with low trough serum infliximab levels and presence of anti-infliximab antibody in maintenance treatment of inflammatory bowel disease (IBD). J Gastroenterol Hepatol 2011;26:56-67.

21. Glossop JR, Dawes PT, Mattey DL. Association between cigarette smoking and release of tumour necrosis factor alpha and its soluble receptors by peripheral blood mononuclear cells in patients with rheumatoid arthritis. Rheumatology (Oxford) 2006;45:1223-1229.

22. Narula N, Fedorak RN. Does smoking reduce infliximab's effectiveness against Crohn's disease? Can J Gastroenterol 2009;23:121-125.

23. Inamdar S, Volfson A, Rosen L, Sunday S, Katz S, Sultan K. Smoking and early infliximab response in Crohn's disease: a meta-analysis. J Crohns Colitis 2015;9:140-146.

24. Dulai PS, Singh S, Jiang X, et al. The real-world effectiveness and safety of vedolizumab for moderate-severe Crohn's disease: results from the US VICTORY Consortium. Am J Gastroenterol 2016;111:1147-1155.

25. Petersen ER, Søndergaard HB, Laursen JH, et al. Smoking is associated with increased disease activity during natalizumab treatment in multiple sclerosis. Mult Scler 2019;25:1298-1305.

26. Hedström AK, Ryner M, Fink K, et al. Smoking and risk of treatment-induced neutralizing antibodies to interferon $\beta$-1a. Mult Scler 2014;20:445-450.

27. Anzengruber F, Augustin M, Radtke MA, et al. Smoking does not alter the therapy response to systemic anti-psoriatic therapies: a two-country, multi-centre, prospective, non-interventional study. Acta Derm Venereol 2019;99:871-877.

28. Umezawa Y, Saeki H, Nakagawa H. Some clinical factors affecting quality of the response to ustekinumab for psoriasis. J Dermatol 2014;41:690-696.

29. Tillack C, Ehmann LM, Friedrich M, et al. Anti-TNF antibodyinduced psoriasiform skin lesions in patients with inflammatory bowel disease are characterised by interferon- $\gamma$-expressing Th1 cells and IL-17A/IL-22-expressing Th17 cells and respond to anti-IL-12/IL-23 antibody treatment. Gut 2014;63:567-577.

30. Sandborn WJ, Su C, Sands BE, et al. Tofacitinib as induction and maintenance therapy for ulcerative colitis. N Engl J Med 2017;376:1723-1736.

31. Karataş A, Öz B, Dalkiliç E, et al. Cigarette smoking does not affect treatment response to tofacitinib in rheumatoid arthritis. Arthritis Rheumatol 2018;70 (Suppl 10):1554.

32. Pfizer. Pfizer announces modification to ongoing tofacitinib FDA post-marketing requirement study in patients with rheumatoid arthritis [Internet]. c2019 [cited 2020 Jan 6]. https://investors.pfizer.com/investor-news/press-release-details/2019/ Pfizer-Announces-Modification-to-Ongoing-Tofacitnib-FDAPost-Marketing-Requirement-Study-in-Patients-with-Rheumatoid-Arthritis/default.aspx.

33. Sandborn WJ, Panés J, Sands BE, et al. Venous thromboembolic events in the tofacitinib ulcerative colitis clinical development programme. Aliment Pharmacol Ther 2019;50:10681076.

34. Winthrop KL, Melmed GY, Vermeire S, et al. Herpes zoster infection in patients with ulcerative colitis receiving tofacitinib. Inflamm Bowel Dis 2018;24:2258-2265.

35. Shen ZH, Zhu CX, Quan YS, et al. Relationship between intestinal microbiota and ulcerative colitis: mechanisms and clinical application of probiotics and fecal microbiota transplantation. World J Gastroenterol 2018;24:5-14.

36. Paramsothy S, Paramsothy R, Rubin DT, et al. Faecal microbiota transplantation for inflammatory bowel disease: a systematic review and meta-analysis. J Crohns Colitis 2017;11:11801199.

37. Sood A, Mahajan R, Singh A, et al. Role of faecal microbiota transplantation for maintenance of remission in patients with ulcerative colitis: a pilot study. J Crohns Colitis 2019;13:13111317. 
38. Lee SH, Yun Y, Kim SJ, et al. Association between cigarette smoking status and composition of gut microbiota: population-based cross-sectional study. J Clin Med 2018;7:282.

39. Biedermann L, Brülisauer K, Zeitz J, et al. Smoking cessation alters intestinal microbiota: insights from quantitative investigations on human fecal samples using FISH. Inflamm Bowel Dis 2014;20:1496-1501.

40. Cosnes J, Nion-Larmurier I, Beaugerie L, Afchain P, Tiret E, Gendre JP. Impact of the increasing use of immunosuppressants in Crohn's disease on the need for intestinal surgery. Gut 2005;54:237-241.

41. Auzolle C, Nancey S, Tran-Minh ML, et al. Male gender, active smoking and previous intestinal resection are risk factors for post-operative endoscopic recurrence in Crohn's disease: results from a prospective cohort study. Aliment Pharmacol Ther 2018;48:924-932.

42. Reese GE, Nanidis T, Borysiewicz C, Yamamoto T, Orchard T, Tekkis PP. The effect of smoking after surgery for Crohn's disease: a meta-analysis of observational studies. Int J Colorectal Dis 2008;23:1213-1221.

43. Kuenzig ME, Lee SM, Eksteen B, et al. Smoking influences the need for surgery in patients with the inflammatory bowel diseases: a systematic review and meta-analysis incorporating disease duration. BMC Gastroenterol 2016;16:143.

44. Gustavsson A, Magnuson A, Blomberg B, Andersson M, Halfvarson J, Tysk C. Smoking is a risk factor for recurrence of intestinal stricture after endoscopic dilation in Crohn's disease. Aliment Pharmacol Ther 2013;37:430-437.

45. Di Stefano A, Capelli A, Lusuardi M, et al. Severity of airflow limitation is associated with severity of airway inflammation in smokers. Am J Respir Crit Care Med 1998;158:1277-1285.

46. MacNee W. Pathogenesis of chronic obstructive pulmonary disease. Proc Am Thorac Soc 2005;2:258-266.

47. Ambrose JA, Barua RS. The pathophysiology of cigarette smoking and cardiovascular disease: an update. J Am Coll Cardiol 2004;43:1731-1737.

48. Berkowitz L, Schultz BM, Salazar GA, et al. Impact of cigarette smoking on the gastrointestinal tract inflammation: opposing effects in Crohn's disease and ulcerative colitis. Front Immunol 2018;9:74.

49. Li LF, Chan RL, Lu L, et al. Cigarette smoking and gastrointestinal diseases: the causal relationship and underlying molecular mechanisms (review). Int J Mol Med 2014;34:372-380.

50. Ueno A, Jijon H, Traves S, et al. Opposing effects of smoking in ulcerative colitis and Crohn's disease may be explained by differential effects on dendritic cells. Inflamm Bowel Dis
2014;20:800-810.

51. Mackern-Oberti JP, Riquelme SA, Llanos C, et al. Heme oxygenase- 1 as a target for the design of gene and pharmaceutical therapies for autoimmune diseases. Curr Gene Ther 2014; 14:218-235.

52. Sher ME, Bank S, Greenberg R, et al. The influence of cigarette smoking on cytokine levels in patients with inflammatory bowel disease. Inflamm Bowel Dis 1999;5:73-78.

53. Lee G, Jung KH, Shin D, et al. Cigarette smoking triggers colitis by IFN- $\gamma+C D 4+T$ cells. Front Immunol 2017;8:1344.

54. Getliffe KM, Al Dulaimi D, Martin-Ruiz C, et al. Lymphocyte telomere dynamics and telomerase activity in inflammatory bowel disease: effect of drugs and smoking. Aliment Pharmacol Ther 2005;21:121-131.

55. Bridger S, Lee JC, Bjarnason I, Jones JE, Macpherson AJ. In siblings with similar genetic susceptibility for inflammatory bowel disease, smokers tend to develop Crohn's disease and non-smokers develop ulcerative colitis. Gut 2002;51:21-25.

56. Nielsen OH, Bjerrum JT, Csillag C, Nielsen FC, Olsen J. Influence of smoking on colonic gene expression profile in Crohn's disease. PLoS One 2009;4:e6210.

57. Opstelten JL, Plassais J, van Mil SW, et al. Gut microbial diversity is reduced in smokers with Crohn's disease. Inflamm Bowel Dis 2016;22:2070-2077.

58. Benjamin JL, Hedin CR, Koutsoumpas A, et al. Smokers with active Crohn's disease have a clinically relevant dysbiosis of the gastrointestinal microbiota. Inflamm Bowel Dis 2012;18: 1092-1100.

59. Kaczynski AT, Manske SR, Mannell RC, Grewal K. Smoking and physical activity: a systematic review. Am J Health Behav 2008;32:93-110.

60. Engels M, Cross RK, Long MD. Exercise in patients with inflammatory bowel diseases: current perspectives. Clin Exp Gastroenterol 2017;11:1-11.

61. Jones PD, Kappelman MD, Martin CF, Chen W, Sandler RS, Long MD. Exercise decreases risk of future active disease in patients with inflammatory bowel disease in remission. Inflamm Bowel Dis 2015;21:1063-1071.

62. Ng V, Millard W, Lebrun C, Howard J. Low-intensity exercise improves quality of life in patients with Crohn's disease. Clin J Sport Med 2007;17:384-388.

63. Loudon CP, Corroll V, Butcher J, Rawsthorne P, Bernstein CN. The effects of physical exercise on patients with Crohn's disease. Am J Gastroenterol 1999;94:697-703.

64. Niewiadomski O, Studd C, Wilson J, et al. Influence of food and lifestyle on the risk of developing inflammatory bowel 
disease. Intern Med J 2016;46:669-676.

65. Xu L, Lochhead P, Ko Y, Claggett B, Leong RW, Ananthakrishnan AN. Systematic review with meta-analysis: breastfeeding and the risk of Crohn's disease and ulcerative colitis. Aliment Pharmacol Ther 2017;46:780-789.

66. MacLean RR, Cowan A, Vernarelli JA. More to gain: dietary energy density is related to smoking status in US adults. BMC Public Health 2018;18:365.

67. Amre DK, D'Souza S, Morgan K, et al. Imbalances in dietary consumption of fatty acids, vegetables, and fruits are associated with risk for Crohn's disease in children. Am J Gastroenterol 2007;102:2016-2025.

68. Ananthakrishnan AN, Khalili H, Konijeti GG, et al. A prospective study of long-term intake of dietary fiber and risk of Crohn's disease and ulcerative colitis. Gastroenterology 2013; 145:970-977.

69. Levine A, Wine E, Assa A, et al. Crohn's disease exclusion diet plus partial enteral nutrition induces sustained remission in a randomized controlled trial. Gastroenterology 2019;157:440450.

70. Muff C, Dragano N, Jöckel KH, et al. Is the co-occurrence of smoking and poor consumption of fruits and vegetables confounded by socioeconomic conditions? Int J Public Health 2010;55:339-346.

71. Sherman BW, Lynch WD. The association of smoking with medical treatment adherence in the workforce of a large employer. Patient Prefer Adherence 2014;8:477-486.

72. Lee J, Jee SR, Kim HW, et al. Factors associated with low adherence to oral 5-aminosalicylic acid in patients with ulcerative colitis. PLoS One 2019;14:e0214129.

73. Lopez A, Billioud V, Peyrin-Biroulet C, Peyrin-Biroulet L. Adherence to anti-TNF therapy in inflammatory bowel diseases: a systematic review. Inflamm Bowel Dis 2013;19:1528-1533.

74. Nielsen MJ, Nørgaard M, Holland-Fisher P, Christensen LA. Self-reported antenatal adherence to medical treatment among pregnant women with Crohn's disease. Aliment Pharmacol Ther 2010;32:49-58.

75. Tribbick D, Salzberg M, Ftanou M, et al. Prevalence of mental health disorders in inflammatory bowel disease: an Australian outpatient cohort. Clin Exp Gastroenterol 2015;8:197204.

76. Porcelli P, Zaka S, Centonze S, Sisto G. Psychological distress and levels of disease activity in inflammatory bowel disease. Ital J Gastroenterol 1994;26:111-115.

77. Mittermaier C, Dejaco C, Waldhoer T, et al. Impact of depressive mood on relapse in patients with inflammatory bowel disease: a prospective 18-month follow-up study. Psychosom Med 2004;66:79-84.

78. Mikocka-Walus A, Pittet V, Rossel JB, von Känel R; Swiss IBD Cohort Study Group. Symptoms of depression and anxiety are independently associated with clinical recurrence of inflammatory bowel disease. Clin Gastroenterol Hepatol 2016; 14:829-835.

79. Sobell LC, Sobell MB, Agrawal S. Self-change and dual recoveries among individuals with alcohol and tobacco problems: current knowledge and future directions. Alcohol Clin Exp Res 2002;26:1936-1938.

80. De Leon J, Rendon DM, Baca-Garcia E, et al. Association between smoking and alcohol use in the general population: stable and unstable odds ratios across two years in two different countries. Alcohol Alcohol 2007;42:252-257.

81. Rajabi A, Dehghani M, Shojaei A, Farjam M, Motevalian SA. Association between tobacco smoking and opioid use: a meta-analysis. Addict Behav 2019;92:225-235.

82. Persaud R. Smokers' rights to health care. J Med Ethics 1995; 21:281-287.

83. Siu AL; U.S. Preventive Services Task Force. Behavioral and pharmacotherapy interventions for tobacco smoking cessation in adults, including pregnant women: U.S. Preventive Services Task Force Recommendation Statement. Ann Intern Med 2015;163:622-634.

84. Babb S, Malarcher A, Schauer G, Asman K, Jamal A. Quitting smoking among adults: United States, 2000-2015. MMWR Morb Mortal Wkly Rep 2017;65:1457-1464.

85. Biedermann L, Fournier N, Misselwitz B, et al. High rates of smoking especially in female Crohn's disease patients and low use of supportive measures to achieve smoking cessation: data from the Swiss IBD cohort study. J Crohns Colitis 2015;9: 819-829.

86. Stephens WE. Comparing the cancer potencies of emissions from vapourised nicotine products including e-cigarettes with those of tobacco smoke. Tob Control 2018:27:10-17.

87. Hartmann-Boyce J, McRobbie H, Bullen C, Begh R, Stead LF, Hajek P. Electronic cigarettes for smoking cessation. Cochrane Database Syst Rev 2016;9:CD010216.

88. McNeill A, Brose LS, Calder R, Bauld L, Robson D. Evidence review of e-cigarettes and heated tobacco products 2018: a report commissioned by Public Health England. London: Public Health England, 2018.

89. Hajek P, Phillips-Waller A, Przulj D, et al. A randomized trial of e-cigarettes versus nicotine-replacement therapy. N Engl J Med 2019;380:629-637. 
90. Bullen C, Howe C, Laugesen M, et al. Electronic cigarettes for smoking cessation: a randomised controlled trial. Lancet 2013;382:1629-1637.

91. Caponnetto P, Campagna D, Cibella F, et al. EffiCiency and Safety of an eLectronic cigAreTte (ECLAT) as tobacco cigarettes substitute: a prospective 12-month randomized control design study. PLoS One 2013;8:e66317.

92. Al-Delaimy WK, Myers MG, Leas EC, Strong DR, Hofstetter CR. E-cigarette use in the past and quitting behavior in the future: a population-based study. Am J Public Health 2015;105: 1213-1219.

93. Grana RA, Popova L, Ling PM. A longitudinal analysis of electronic cigarette use and smoking cessation. JAMA Intern Med 2014;174:812-813.

94. Werner AK, Koumans EH, Chatham-Stephens K, et al. Hospi- talizations and deaths associated with EVALI. N Engl J Med 2020;382:1589-1598.

95. Katz MG, Russell KW. Injury from e-cigarette explosion. N Engl J Med 2019;380:2460.

96. Layden JE, Ghinai I, Pray I, et al. Pulmonary illness related to e-cigarette use in Illinois and Wisconsin: final report. N Engl J Med 2020;382:903-916.

97. Stewart CJ, Auchtung TA, Ajami NJ, et al. Effects of tobacco smoke and electronic cigarette vapor exposure on the oral and gut microbiota in humans: a pilot study. PeerJ 2018;6: e4693.

98. Chong C, Rahman A, Loonat K, Sagar RC, Selinger CP. Current smoking habits in British IBD patients in the age of e-cigarettes. BMJ Open Gastroenterol 2019;6:e00309. 